OPEN

SUBJECT AREAS:

INFORMATION THEORY AND COMPUTATION

QUANTUM PHYSICS

APPLIED PHYSICS

MATHEMATICS

Received

2 July 2012

Accepted

1 August 2012

Published

22 August 2012

Correspondence and requests for materials should be addressed to A.T.S. (ats@math.uga. edu)

\section{Quantum Simulation of Tunneling in Small Systems}

\author{
Andrew T. Sornborger
}

Department of Mathematics and College of Engineering University of Georgia, Athens, Georgia 30602, USA.

A number of quantum algorithms have been performed on small quantum computers; these include Shor's prime factorization algorithm, error correction, Grover's search algorithm and number of analog and digital quantum simulations. Because of the number of gates and qubits necessary, however, digital quantum particle simulations remain untested. A contributing factor to the system size required is the number of ancillary qubits needed to implement matrix exponentials of the potential operator. Here, we show that a set of tunneling problems may be investigated with no ancillary qubits and a cost of one single-qubit operator per time step for the potential evolution, eliminating at least half of the quantum gates required for the algorithm and more than that in the general case. Such simulations are within reach of current quantum computer architectures.

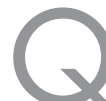

uantum simulations on quantum computers are one of a set of algorithms that give exponential improvement in computational resources relative to the best classical algorithm ${ }^{1-4}$.

Algorithms for studying many types of quantum field theory have been considered in both analog form in which a quantum Hamiltonian (typically manybody or multiple spin) is mapped either directly or via a suitable pulse-sequence to a computational Hamiltonian ${ }^{5-21}$ and digital form in which a quantum system's Hamiltonian is split into free and interacting operators, then, using Trotter's formula, is simulated on a quantum computer ${ }^{22,23}$.

Small quantum simulations have already been realized on $\mathrm{NMR}^{24-31}$, atomic ${ }^{32,33}$, ion trap ${ }^{34-38}$ and photonic ${ }^{39,40}$ quantum computers in both analog and digital forms.

A concerted effort has also been made to investigate digital quantum particle simulations ${ }^{3}$ for the simulation of chemical dynamics ${ }^{41,42}$. Algorithms for state preparation ${ }^{43}$, the simulation of temporal dynamics ${ }^{41}$ and the measurement of observables ${ }^{44}$ have all been developed. However, this type of simulation has remained untested due to the large number of gates and/or ancillary qubits needed to compute the kinetic and potential operators ${ }^{41,45}$. In this paper, we focus on reducing the number of gates required for the simulation of temporal dynamics to the bare minimum, while still simulating interesting physics.

For a review of the current state and outlook for quantum simulation, see ${ }^{46-50}$ and $^{51}$.

The standard digital quantum simulation algorithm for a particle on a one-dimensional grid ${ }^{3,45}$ encodes the position efficiently in $n=\log _{2} N$ qubits, where $N$ is the size of the lattice of discretized particle locations, $x_{k}=k \Delta x$, $k=0, \ldots, N-1$. The method uses a split operator approach to integrate a Schrödinger equation with a timeindependent Hamiltonian that is first-order accurate in the time step, $\Delta t^{3}$ :

$$
\begin{aligned}
|\psi(t)\rangle & =e^{-i H t}\left|\psi_{\text {init }}\right\rangle \\
& =e^{-i(V+K) t}\left|\psi_{\text {init }}\right\rangle \\
& =\left(e^{-i V \Delta t} e^{-i K \Delta t} e^{O\left(\Delta t^{2}\right)}\right)^{\frac{t}{\Delta t}}\left|\psi_{\text {init }}\right\rangle .
\end{aligned}
$$

Higher order methods that give more accurate time integration have been developed ${ }^{52-54}$, but methods of order higher than two require more gates per time step. For simplicity, we will only consider first-order methods here. States in the qubit Hilbert space

$$
|\psi\rangle=\left|\ldots \psi_{2} \psi_{1} \psi_{0}\right\rangle=\ldots\left|\psi_{2}\right\rangle\left|\psi_{1}\right\rangle\left|\psi_{0}\right\rangle
$$

where $\left|\psi_{i}\right\rangle \in\{|0\rangle,|1\rangle\}$, represent particle location in the binary representation, $|x\rangle=\Sigma_{j \in 0, \ldots, n-1} 2^{j} \psi_{j}|\psi\rangle$. The matrix exponential for the kinetic operator is calculated using a quantum Fourier transform (QFT) 

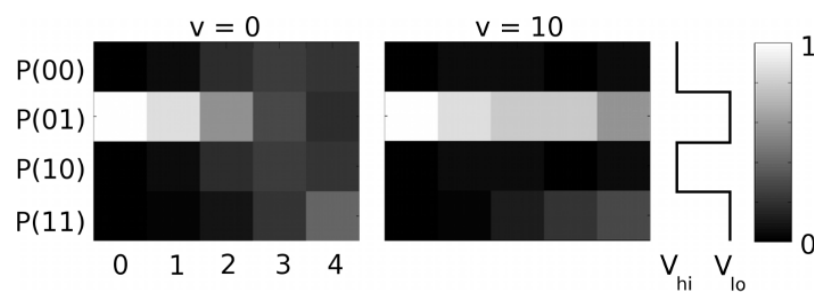

Figure $1 \mid$ Particle probability distributions as a function of time for the first four steps of a two qubit simulation for $v=0$ (free particle) and $v=$ 10 (particle in double well). The double-well potential and gray scale used to plot the probabilities are depicted to the right.

$$
e^{-i K t}=F e^{-i T t} F^{\dagger},
$$

where $F^{\dagger}$ is a discrete Fourier transform operator and $T$ is diagonal with entries proportional to $-q^{2} / 2 m$, and $q$ denotes the Fourier mode wavenumber. The resulting periodic, shift invariant unitary transform, $e^{-i K \Delta t}$, gives an approximation to the time evolution on the lattice due to the kinetic energy operator that is accurate to $N$ th order in space. This leads to the digital quantum particle simulation algorithm:

$$
|\psi(t)\rangle:=\left(e^{-i V \Delta t} F e^{-i T \Delta t} F^{\dagger}\right)^{\frac{t}{\Delta t}}|\psi(0)\rangle .
$$

The QFT takes of order $n^{2}$ gates to calculate ${ }^{55}$ and general algorithms implementing the diagonal $T$ and $V$ operators require ancillary qubits ${ }^{56}$, although it has been shown that the quadratic kinetic energy operator may be computed with $n^{2}$ two-qubit gates with no ancillary qubits $^{45}$.

The point of this paper is to describe a quantum particle simulation that can be used as a proof-of-principle demonstration. To do this, it must be implemented in current quantum computer architectures where the number of qubits is limited and the number of gates that may be performed before decoherence destroys the computation is also limited. We consider the special case of square-well potentials. We show that a set of square-well potentials may be implemented with a sole, single-qubit operator and no ancillary qubits (see Methods). This virtually eliminates the calculation of the potential operator. Thus, simulations of important physical phenomena such as tunneling and the evolution of quasi-stable states may be performed with considerably fewer gates than simulations requiring arbitrary potentials. Additionally, we present a reduction of the number of gates required for the diagonal part of the kinetic operator in the case of 4 or fewer qubits.

\section{Results}

A Two Qubit Tunneling Simulation. Let us consider the smallest possible tunneling simulation. An $n=2$ qubit simulation of $N=4$ lattice points may be performed with the circuit

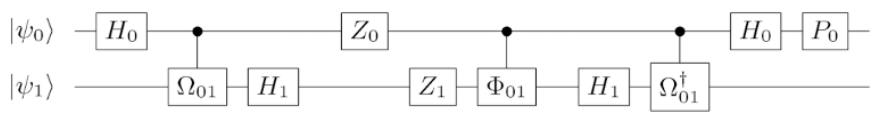

One Time Step of a Two-qubit Digital Quantum Single-particle Tunneling Simulation.

In this circuit and below, $\Omega_{i j}$ and $\Phi_{i j}$ are controlled phase gates applied to qubits $i$ and $j$ and $H_{i}$ and $Z_{i}$ are a Hadamard gate and a $Z$ rotation on qubit $i$, respectively (see Methods). In total, each time step in this tunneling simulation requires 10 operations, 7 single qubit operations and 3 two-qubit operations. The circuit for a single time step is shown above. This circuit implements a double-well potential with the gate $P_{0}=\exp \left(-i v \sigma_{z}^{0} \Delta t\right)$ acting on the lowest order qubit.
In Fig. 1, we plot lattice occupation probabilities from two simulations with a double-well potential with $\Delta t=1 / 10$ : one a free-particle simulation with $v=0$ and the other a tunneling simulation with $v=$ 10. This value of $\Delta t$ traded off accuracy for gate number (see Methods section for a discussion of simulation errors), but the qualitative dynamics did not change even for more accurate (and costly) simulations. The initial state was $\left|\psi_{\text {initi }}\right\rangle=|01\rangle$, corresponding to a particle in one of the wells. The free-particle probability distribution spreads across all lattice points as it evolves, whereas the particle tunnels from the well at lattice point $1(|01\rangle)$ to the well at lattice point $3(|11\rangle)$ in the tunneling simulation. These results show that differences in the evolution of the probability distribution are evident within 4 time steps. Thus, such a proof-of-principle simulation may be implemented on a quantum computer with $4 \times 10=40$ gates $(28$ single-qubit and 12 two-qubit).

Multi-Qubit Quantum Tunneling Simulations. Larger simulations require more gates. For instance, a three-qubit simulation requires 6 gates per QFT (3 singlequbit and 3 two-qubit), 6 gates for the diagonal kinetic energy operator ( 3 singlequbit and 3 two-qubit), and one single-qubit gate for the potential as shown in the circuit diagram below.

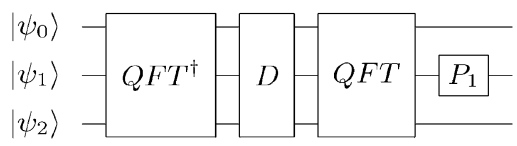

where $P_{1}=\exp \left(-i v \sigma_{z}^{1} \Delta t\right)$ is shown, representatively, for a doublewell potential, but other square-well potentials could be generated by acting on different qubits. The QFT and diagonal kinetic energy operators are

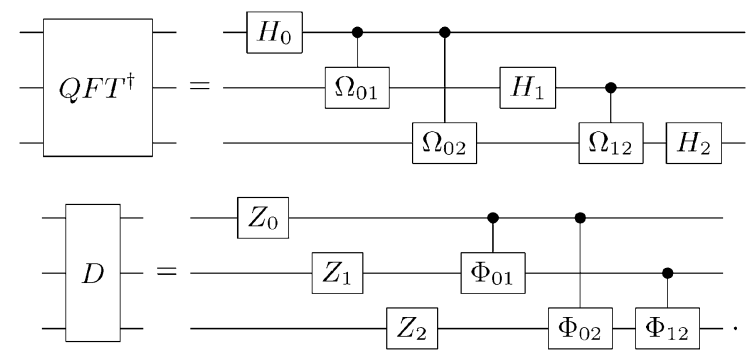

In Fig. 2, we show results from a three-qubit simulation with a double-well potential, where each well is resolved with two lattice points. The time step $\Delta t=1 / 5$ and $v=5$. Here, a tradeoff was also necessary to find a simulation that captured the oscillatory and tunneling time scales and had few gates. The initial state was $\left|\psi_{\text {initi }}\right\rangle=$ $|110\rangle$. Because the initial state only occupies half of one well, oscillatory dynamics are visible within the well. After a few time steps, the oscillatory state tunnels between wells. Oscillatory dynamics are evident within 4 time steps, but 5 time steps are required before the oscillatory state tunnels appreciably to the second well and 7 time steps are needed to see oscillation of the tunneled state. Thus, between $4 \times 19=76$ or $7 \times 19=133$ gates would be required in order to see interesting tunneling effects in such a simulation. Fourqubit simulations can be envisaged using circuits based on similar methodology, although the number of gates would most likely be prohibitive for current quantum computer architectures.

\section{Discussion}

Only $n=\log _{2} N$ qubits are required for an $N$ lattice-point particle simulation, therefore this algorithm is efficient in the number of qubit resources required. In general, $n^{2}$ gates are needed for the kinetic operator (see Methods), but for fewer than $n=4$ qubits, 


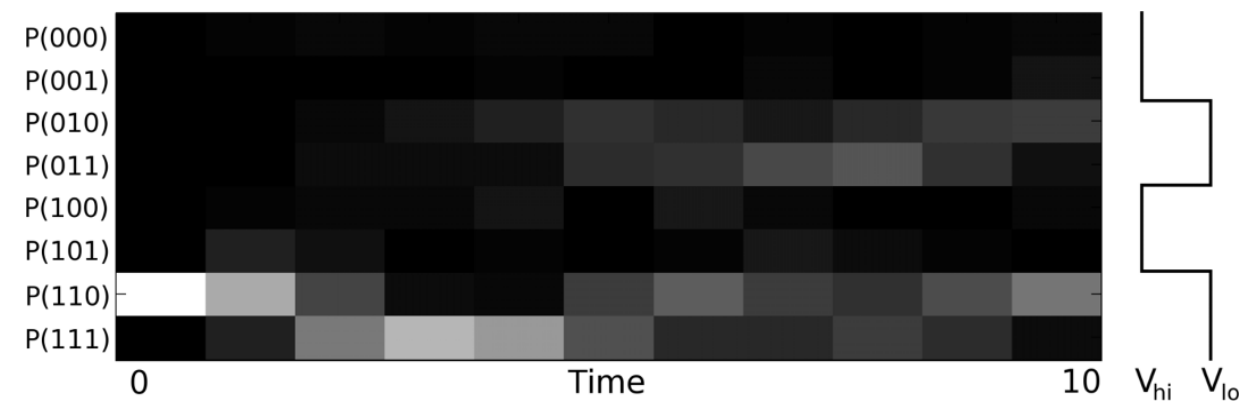

Figure $2 \mid$ Particle probability distribution as a function of time over ten time steps in a three-qubit double-well simulation. The potential schematic is shown to the right, as in Fig. 1. Gray scales are as in Fig. 1. The double-well potential has two lattice points per well and, for the given initial state, an oscillation is induced in one of the wells then tunnels to the other well.

further efficiency is possible using the basis approach that we outlined. Furthermore, as noted above, our algorithm virtually eliminates the calculation of the potential operator. With very few qubits, interesting tunneling dynamics may be simulated with a gate count that is within reach of current quantum architectures.

Much recent work has been done to understand the resources necessary to perform fully error-corrected quantum particle simulations using various error correction schemes ${ }^{57}$. We note that, as in the non-fault-tolerant case, the simulations presented here would also require the fewest resources in fully error corrected quantum architectures, since the number of logical qubits and gates is as small as possible in the error-corrected case as well.

\section{Methods}

Square-well Potentials. A set of square-well potentials may be implemented with a sole single-qubit operator and no ancillary qubits. To see this, consider the singlequbit $Z$-rotation on the highest order qubit

$$
e^{-i V \Delta t}=e^{-i v \sigma_{z}^{n-1} \Delta t}=e^{-i v \sigma_{z} \Delta t} \otimes I \otimes I \ldots,
$$

where $v$ is a parameter, a superscript indicates the qubit to which the operator is applied and $\sigma_{z}$ is the Pauli $z$-matrix $\sigma_{z}=\left(\begin{array}{cc}1 & 0 \\ 0 & -1\end{array}\right)$. The operator $e^{-i V \Delta t}$, when acting on a lattice state, implements a square-well potential by rotating qubit states with $|0\rangle$ (|1 $\rangle$ resp.) highest order qubit with positive (negative resp.) phase velocity $v$. The single-qubit operator acting on the next highest order qubit

$$
e^{-i V \Delta t}=e^{-i v \sigma_{z}^{n-2} \Delta t}=I \otimes e^{-i v \sigma_{z} \Delta t} \otimes I \ldots
$$

implements a double-square-well potential, and so on, with the last potential in this series implementing a Dirac comb-like potential.

By simulating this class of square-well potentials we reduce the complexity of the potential calculation from a potentially large number of gates and ancillary qubits to just one single-qubit operation. In contrast, the phase kickback algorithm used in $^{41}$ requires a total of $2 n$ qubits ( $n$ for the quantum state and $n$ ancilla qubits) as well as $n^{2}$ extra gates for a QFT on $n$ ancilla qubits.

Although the main point of this paper is the simplification of the simulation by reducing the potential computation to one gate, further gate reductions can be made to the kinetic operator when considering few qubit systems. This can be done by forming a diagonal Hamiltonian using a basis of diagonal operators. This method is only efficient for $n \leq 4$ and scales as $2^{n}-1$. Therefore, for $n>4$, ancillary qubits or the Benenti-Strini (BS $)^{45}$ method (that scales as $n^{2}$ ) should be used. However, for small systems of qubits, forming a basis of diagonal operators takes fewer gates. Note that for $n=2,3$ and 4, we would need 4, 9 and 16 (resp.) operators for BS, but at most 3, 7 and 15 (resp.) are required using a basis along the diagonal. Furthermore, fewer multiqubit operations are necessary with this method, although a three-qubit operator may be necessary for three-qubit simulations and three- and four-qubit operators for fourqubit simulations. Here, we use the method to construct the diagonal operator $D$ for the kinetic energy operator, however, arbitrary potentials could also be constructed this way. Because our goal is to find interesting simulations with few gates, we do not pursue more complex potentials here.

In our two-qubit simulations (see Results), we use a double well potential, $\exp (-i V \Delta t)=\exp \left(-i v \sigma_{z}^{0} \Delta t\right)$. The QFT may be computed with the operators ${ }^{55}$

$$
F^{\dagger}=H_{1} \Omega_{01} H_{0},
$$

where $H_{i}$ is a Hadamard operator on the $i$ th qubit and the controlled-phase gate $\Omega_{01}$ $=\operatorname{diag}(1,1,1, \omega)$ with $\omega=\exp (2 \pi i / 4)$. Note that $F^{\dagger}$ results in a bitswapped Fourier transform (in our notation, $F$ is the inverse Fourier transform matrix).
The kinetic operator is then $K=F D F^{\dagger}$, with

$$
D=\exp \left(-i(-2 \pi / 4)^{2} \operatorname{diag}(0,4,1,1) \Delta t\right)
$$

(Note that this operator is also bit-swapped and we have taken $m=1 / 2$ ).

The diagonal operation $D$ may be achieved up to an overall phase with the operator

$$
D=\Phi_{01} Z_{1} Z_{0}
$$

where the single-qubit operators

$$
\begin{aligned}
& Z_{0}=e^{-i \gamma c_{0} \sigma_{z}^{0} \Delta t} \\
& Z_{1}=e^{-i \gamma c_{1} \sigma_{z}^{1} \Delta t}
\end{aligned}
$$

and $\Phi_{01}$ is a controlled-phase operator on qubits 0 and 1 ,

$$
\Phi_{01}=e^{-i \gamma c_{2} \operatorname{diag}(1,1,1,-1)_{01} \Delta t},
$$

where $\gamma=(-2 \pi / 4)^{2} / \sqrt{4}$. The coefficients $\left(c_{0}=-1, c_{1}=-4\right.$ and $\left.c_{2}=4\right)$ in the unitary operators $Z_{0}, Z_{1}$ and $\Phi_{01}$ (resp.) were obtained by noting that the vectors $(1,1,-1,-1)$, $(1,-1,1,-1)$ and $(1,1,1,-1)$ that form their diagonal elements are a basis for zero mean vectors (i.e. neglecting the constant phase proportional to $(1,1,1,1))$ for $\mathbb{R}^{4}$.

To calculate the QFT in the three-qubit case, the unitary operators are the single-qubit Hadamard gates and the two-qubit controlled-phase operators $\Omega_{i j}=\Omega_{i j}(\omega)$, where $\omega=$ $\exp (2 \pi i / 8)$ (see circuit diagram in Results). To calculate the quadratic diagonal operator in the three-qubit case (see Results), the unitary operators are the single-qubit operators

$$
\begin{aligned}
& Z_{0}=\exp \left(-i \gamma c_{0} \sigma_{z}^{0} \Delta t\right) \\
& Z_{1}=\exp \left(-i \gamma c_{1} \sigma_{z}^{1} \Delta t\right) \\
& Z_{2}=\exp \left(-i \gamma c_{2} \sigma_{z}^{2} \Delta t\right)
\end{aligned}
$$

and the two-qubit controlled-phase operators

$$
\begin{aligned}
& \Phi_{01}=\exp \left(-i \gamma c_{3} \operatorname{diag}(1,1,1,-1)_{01} \Delta t\right) \\
& \Phi_{02}=\exp \left(-i \gamma c_{4} \operatorname{diag}(1,1,1,-1)_{02} \Delta t\right) \\
& \Phi_{12}=\exp \left(-i \gamma c_{5} \operatorname{diag}(1,1,1,-1)_{12} \Delta t\right),
\end{aligned}
$$

where

$$
\gamma=-(2 \pi / 8)^{2} / \sqrt{8}
$$

and

$$
\begin{aligned}
& c_{0}=-1.42 \\
& c_{1}=-5.66 \\
& c_{2}=-22.63 \\
& c_{3}=22.63 \\
& c_{4}=11.31 \\
& c_{5}=-5.66 .
\end{aligned}
$$

Note that, in principle, a seventh three-qubit operator would also be necessary propor tional to the vector $(1,1,1,1,1,1,1,-1)$ along the diagonal, but for the (bit-swapped) diagonal of the 8 lattice point simulation, $(0,16,4,4,1,9,9,1)$, its coefficient is identically zero. This circuit requires a total of 19 gates (10 single-qubit and 9 two-qubit).

Once we have computed the unitary operators for one time step of the kinetic, $U_{k}(\Delta t)$, and potential, $U_{v}(\Delta t)$, evolution, it is straightforward to calculate the exact 
Table 1 | RMS Error per time step for a range of values of $\Delta t$ for the 2 and 3 qubit simulations described in Results

\begin{tabular}{lccccl|}
$\Delta \mathrm{t}$ & 0.2 & 0.1 & 0.05 & 0.025 & 0.0125 \\
\hline Error 2 qubit & 0.40 & 0.16 & 0.06 & 0.03 & 0.01 \\
Error 3 qubit & 0.08 & 0.02 & 0.007 & 0.003 & 0.002 \\
\hline
\end{tabular}

Hamiltonian evolution: $U_{\text {exact }}(\Delta t)=\exp \left(\log \left(U_{k}(\Delta t)\right)+\log \left(U_{v}(\Delta t)\right)\right)$. We then determine RMS errors relative to the exact evolution for the two and three-qubit simulations. These are given in Table 1. The simulations that we present in Figs. 1 and 2 have a total, integrated error of $64 \%$ and $20 \%$, respectively. For both simulations, we checked that state evolution was qualitatively similar to the exact simulations for the chosen values of $\Delta t$. Clearly, for high-precision simulations, smaller errors would be desirable. To achieve this, one would use smaller values for $\Delta t$ at the expense of an increase in gate count.

1. Lloyd, S. Universal quantum simulators. Science 273, 1073-1078 (1996).

2. Abrams, D. S. \& Lloyd, S. Simulation of many-body Fermi systems on a universal quantum computer. Phys. Rev. Lett. 79, 2586-2589 (1997).

3. Zalka, C. Simulating quantum systems on a quantum computer. Proc. R. Soc. Lond. A 454, 313-322 (1998).

4. Berry, D. et al. Efficient quantum algorithms for simulating sparse Hamiltonians. Commun. Math. Phys. 270, 359-371 (2007).

5. Büchler, H. P. et al. Atomic quantum simulator for lattice gauge theories and ring exchange models. Phys. Rev. Lett. 95, 040402 (2005).

6. Zohar, E. \& Reznik, B. Confinement and lattice quantum-electrodynamic electric flux tubes simulated with ultracold atoms. Phys. Rev. Lett. 107, 275301 (2011).

7. Szirmai, G. et al. Gauge fields emerging from time reversal symmetry breaking for spin-5/2 fermions in a honeycomb lattice. Phys. Rev. A 84, 011611(R) (2011).

8. Cirac, J. I. et al. Cold atom simulation of interacting relativistic quantum field theories. Phys. Rev. Lett. 105, 190403 (2010).

9. Mazza, L. et al. An optical-lattice-based quantum simulator for relativistic field theories and topological insulators. New J. Phys. 14, 015007 (2012).

10. Kapit, E. \& Mueller, E. Optical-lattice Hamiltonians for relativistic quantum electrodynamics. Phys. Rev. A 83, 033625 (2011).

11. Bermudez, A. et al. Wilson fermions and axion electrodynamics in optical lattices. Phys. Rev. Lett. 105, 190404 (2010).

12. Maraner, P. \& Pachos, J. K. Yang-Mills gauge theories from simple fermionic lattice models. Phys. Lett. A 373, 2542 (2009).

13. Lepori, L. et al. $(3+1)$ massive Dirac fermions with ultracold atoms in frustrated cubic optical lattices. Europhys. Lett. 92, 50003 (2010).

14. Maeda, K. et al. Simulating dense QCD matter with ultracold atomic bosonfermion mixtures. Phys. Rev. Lett. 103, 085301 (2009).

15. Rapp, Á. et al. Color superfluidity and "baryon" formation in ultracold fermions. Phys. Rev. Lett. 98, 160405 (2007).

16. Weimer, H. et al. A Rydberg quantum simulator. Nat. Phys. 6, 382 (2010).

17. Casanova, J. et al. Quantum simulation of quantum field theories in trapped ions. Phys. Rev. Lett. 107, 260501 (2011).

18. Casanova, J. et al. Quantum simulation of interacting fermion lattice models in trapped ions. ArXiv 1110.3730 (2011).

19. Douçot, B. et al. Discrete non-Abelian gauge theories in Josephson junction arrays and quantum computation. Phys. Rev. B 69, 214501 (2004).

20. Lewenstein, M. et al. Ultracold atomic gases in optical lattices: Mimicking condensed matter physics and beyond. Adv. Phys. 56, 243-379 (2007)

21. Johanning, M. et al. Quantum simulations with cold trapped ions. J. Phys. B 42, 154009 (2009).

22. Jordan, S. P. et al. Quantum algorithms for quantum field theories. Science 336, $1130-1133$ (2012).

23. Byrnes, T. \& Yamamoto, Y. Simulating lattice gauge theories on a quantum computer. Phys. Rev. A 73, 022328 (2006).

24. Tseng, C. H. et al. Quantum simulation of a three-body-interaction Hamiltonian on an NMR quantum computer. Phys. Rev. A 61, 012302 (1999).

25. Somaroo, S. et al. Quantum simulations on a quantum computer. Phys. Rev. Lett. 82, 5381-5383 (1999).

26. Khitrin, A. K. \& Fung, B. M. NMR simulation of an eight-state quantum system. Phys. Rev. A 64, 032306 (2001).

27. Negrevergne, C. et al. Liquid-state NMR simulations of quantum many body problems. Phys. Rev. A 71, 032344 (2005).

28. Peng, X. H. et al. Quantum phase transition of ground-state entanglement in a Heisenberg spin chain simulated in an NMR quantum computer. Phys. Rev. A 71, 012307 (2005).
29. Brown, K. R. et al. Limitations of quantum simulation examined by a pairing Hamiltonian using nuclear magnetic resonance. Phys. Rev. Lett. 97, 050504 (2006).

30. Peng, X. H. et al. Quantum simulation of a system with competing two and threebody interactions. Phys. Rev. Lett. 103, 140501 (2009).

31. Du, J. F. et al. NMR implementation of a molecular hydrogen quantum simulation with adiabatic state preparation. Phys. Rev. Lett. 104, 030502 (2010).

32. Edwards, E. E. et al. Quantum simulation and phase diagram of the transverse field Ising model with three atomic spins. Phys. Rev. B 82, 060412 (2010).

33. Kinoshita, T. et al. Observation of a one-dimensional Tonks-Girardeau gas. Science 305, 1125-1128 (2004).

34. Friedenauer, A. et al. Simulating a quantum magnet with trapped ions. Nature Physics 4, 757-761 (2008).

35. Gerritsma, R. et al. Quantum simulation of the Dirac equation. Nature 463, 68-71 (2010).

36. Gerritsma, R. et al. Quantum simulation of the Klein paradox with trapped ions. Phys. Rev. Lett. 106, 060503 (2011).

37. Lanyon, B. P. et al. Universal digital quantum simulation with trapped ions. Science 334, 57-61 (2011).

38. Lanyon, B. P. et al. Towards quantum chemistry on a quantum computer. Nature Chemistry 2, 106-111 (2010).

39. Ma, X. S. et al. Quantum simulation of the wavefunction to probe frustrated Heisenberg spin systems. Nature Physics 7, 399-405 (2011).

40. Kassal, I. et al. Simulating chemistry using quantum computers. Annu. Rev. Phys. Chem. 62, 185-207 (2011).

41. Kassal, I. et al. Polynomial-time quantum algorithms for the simulation of chemical dynamics. Proc. Nat. Acad. Sci. USA 105, 18681-18686 (2008).

42. Yung, M.-H. et al. Introduction to quantum algorithms for physics and chemistry. ArXiv:1203.1331 (2012)

43. Ward, N. J. et al. Preparation of many-body states for quantum simulation. J. Chem. Phys. 130, 234113 (2009).

44. Kassal, I. \& Aspuru-Guzik, A. Quantum algorithm for molecular properties and geometry optimization. J. Chem. Phys. 131, 224102 (2009).

45. Benenti, G. \& Strini, G. Quantum simulation of the single-particle Schrödinger equation. Am. J. Phys. 76, 657-662 (2008).

46. Cirac, J. I. \& Zoller, P. Goals and opportunities in quantum simulation. Nature Physics 8, 264-266 (2012).

47. Bloch, I. et al. Quantum simulations with ultracold quantum gases. Nature Physics 8, 267-276 (2012).

48. Blatt, R. \& Roos, C. F. Quantum simulations with trapped ions. Nature Physics 8, 277-284 (2012)

49. Aspuru-Guzik, A. \& Walther, P. Photonic quantum simulators. Nature Physics $\mathbf{8}$, 285-291 (2012)

50. Houck, A. A. et al. On-chip quantum simulation with superconducting circuits. Nature Physics 8, 292-299 (2012).

51. Brown, K. L. et al. Using quantum computers for quantum simulations. Entropy 12, 2268-2307 (2010)

52. Yoshida, H. Construction of higher-order symplectic integrators. Phys. Lett. A 150, 262-268 (1990)

53. Sornborger, A. T. \& Stewart, E. D. Higher-order methods for simulations on quantum computers. Phys. Rev. A 60, 1956-1965 (1999).

54. Hatano, N. \& Suzuki, M. Quantum Annealing and Other Optimization Methods. Springer, Heidelberg, 2005.

55. Coppersmith, D. An approximate Fourier transform useful in quantum factoring IBM Research Report, RC 19642, 1994.

56. Childs, A. Quantum information processing in continuous time. Massachusetts Institute of Technology, 2004

57. Jones, N. Cody, et al. Simulating chemistry efficiently on fault-tolerant quantum computers. ArXiv:1204.0567 (2012)

\section{Acknowledgements}

This work was supported by NSF grants PHY 0939853 and DMS 1029764. The author would like to thank Mike Geller and Phillip Stancil in the Physics Department at the University of Georgia for helpful comments and discussions.

\section{Additional information}

Competing financial interests: The author declares no competing financial interests.

License: This work is licensed under a Creative Commons

Attribution-NonCommercial-No Derivative Works 3.0 Unported License. To view a copy of this license, visit http://creativecommons.org/licenses/by-nc-nd/3.0/

How to cite this article: Sornborger, A.T. Quantum Simulation of Tunneling in Smal Systems. Sci. Rep. 2, 597; DOI:10.1038/srep00597 (2012). 